\title{
Community-acquired pneumonia in outpatients: aetiology and outcomes
}

\author{
Catia Cillóniz*,\#, Santiago Ewig", Eva Polverino*,\#, Maria Angeles Marcos, \\ Elena Prina ${ }^{\S}$, Jacobo Sellares, ${ }^{\star *}$, Miquel Ferrer*, ${ }^{\star}$, Mar Ortega ${ }^{\dagger}$, Albert Gabarrús*,\#, \\ Josep Mensa ${ }^{f}$ and Antoni Torres**\#
}

ABSTRACT: The purpose of this study was to establish the microbial aetiology and outcomes of patients with community-acquired pneumonia (CAP) treated as outpatients after presenting to a hospital emergency care unit.

A prospective observational study was carried out in the Hospital Clinic of Barcelona (Barcelona, Spain). All consecutive cases of CAP treated as outpatients were included.

568 adult outpatients with CAP were studied (mean \pm SD age $47.2 \pm 17.6$ yrs; 110 (19.4\%) were aged $\geqslant 65 \mathrm{yrs}$ ). Aetiological diagnoses were established in $188(33.1 \%)$ cases. Streptococcus pneumoniae was the most frequent pathogen followed by Mycoplasma pneumoniae and respiratory viruses. Legionella was detected in $13(2.3 \%)$ cases. More than one causative agent was found in $17(9.0 \%)$ patients. Mortality was low (three $(0.5 \%)$ patients died) and other adverse events were rare $(30(5.2 \%)$ patients had complications, $13(2.3 \%)$ were re-admitted and treatment failed in $13(2.3 \%)$ ). Complications were mostly related to pleural effusion and empyema, and readmissions and treatment failures to comorbidities.

Outpatients with CAP have a characteristic microbial pattern. Regular antipneumococcal coverage remains mandatory. Treatment failures and re-admissions are rare and may be reduced by increased attention to patients requiring short-term observation in the emergency care unit and in the presence of pleural effusion and comorbidities.

KEYWORDS: Community-acquired pneumonia, outpatients, respiratory infection

$\mathrm{n}$ both the USA and Europe, communityacquired pneumonia (CAP) is the most frequent cause of death due to infection and has considerable implications for healthcare systems worldwide [1-3].

Selection of the initial site of care is one of the most important clinical decisions made in the treatment of CAP and directly affects the intensity of laboratory testing, microbiological evaluation and antibiotic therapy [4]. The microbiology and outcomes of hospitalised patients with CAP is very well known [5-7]. A variety of severity assessment tools have been developed for the identification of patients at low risk in order to guide decisions on hospitalisation [8, 9]. Previous studies have been performed outside the hospital [10-12]. Surprisingly, there are no data from patients visiting the emergency department, and treated as outpatients and followed up for 1 month.
The purpose of this study was to establish the aetiology and outcomes of a large series of nonhospitalised patients with CAP who initially visited the emergency department.

\section{METHODS}

\section{Study setting and design}

Prospective observational study carried out in the Hospital Clinic of Barcelona (Barcelona, Spain), an 800-bed, third-level hospital covering an urban population of 540,000 inhabitants. All consecutive cases of CAP visiting the emergency department and treated as outpatients from January 2000 to July 2010 were included.

\section{Study population}

The study population consisted of adults aged $\geqslant 16$ yrs consecutively admitted to the emergency department with a diagnosis of CAP and discharged for ambulatory treatment. Pneumonia was

\section{AFFILIATIONS}

*Dept of Pneumology, Institut del Tórax, Hospital Clinic, IDIBAPS University of Barcelona, ${ }^{f}$ Dept of Infectious Diseases, Hospital Clinic, IDIBAPS *Centro de Investigación Biomédica En Red-Enfermedades Respiratorias (CibeRes, CB06/06/0028), and +Dept of Microbiology, Hospital Clinic of Barcelona, Barcelona, Spain.

"Thoraxzentrum Ruhrgebiet, Kliniken für Pneumologie und Infektiologie, EVK Herne und Augusta-KrankenAnstalt, Bochum, Germany ${ }^{\S}$ Dipartimento di Medicina d'Urgenza, Policlinico - Universitá degli Studi di Milano, Milan, Italy.

CORRESPONDENCE

A. Torres

Dept of Pneumology

Hospital Clinic

Villarroel 170

08036 Barcelona

Spain

E-mail: atorres@ub.edu

Received:

Sept 292011

Accepted after revision:

Dec 302011

First published online:

Jan 202012 
defined in the presence of a new infiltrate on the chest radiograph (evaluated by the attending physician prior to deciding treatment and site of care), together with symptoms suggestive of a lower respiratory tract infection and the absence of an alternative diagnosis during follow-up. Exclusion criteria were: severe immunosuppression, such as in solid-organ or bonemarrow transplantation or AIDS, or receiving chemotherapy or other immunosuppressive drugs ( $>20 \mathrm{mg}$ prednisone-equivalent per day for $\geqslant 2$ weeks); active tuberculosis; healthcareassociated pneumonia; and cases with a confirmed alternative diagnosis at the end of follow-up.

This study was approved by the ethical committee of the Hospital Clinic of Barcelona (registration number 2009/5451). Patient data were anonymised and informed consent was waived due to the observational nature of the study.

\section{Data collection and follow-up}

Data collected at the time of hospital admission has been reported previously [6]. All surviving patients were re-examined or at least contacted by telephone $\geqslant 30$ days after discharge from the emergency care unit. Pneumonia Severity Index (PSI) and CURB-65 (confusion of new onset, urea $>7 \mathrm{mmol} \cdot \mathrm{L}^{-1}$, respiratory rate $\geqslant 30$ breaths $\mathrm{min}^{-1}$, blood pressure $<90 \mathrm{mmHg}$ systolic or diastolic blood pressure $\leqslant 60 \mathrm{mmHg}$, and age $\geqslant 65 \mathrm{yrs}$ ) score classes were assigned according to the original authors' designations [8, 9].

\section{Definitions}

Outpatient treatment was defined as discharge from the emergency care observation unit to any outpatient setting or discharge from an emergency care observation unit within $24 \mathrm{~h}$ of presentation [13].

Current smokers were defined as anyone who had at some time smoked at least one cigarette per day or one cigar or pipe per week for $\geqslant 1$ yr; an ex-smoker was defined as a smoker who had given up the habit $\geqslant 1$ yr before the diagnosis of CAP [14]. Alcohol abuse was considered in cases with a current intake of $\geqslant 80 \mathrm{~g}$ of alcohol per day in males and $60 \mathrm{~g}$ per day in females [15].

Appropriateness of empirical antibiotic treatment in all patients was defined according to the treatment guidelines of the Spanish Society of Pulmonology and Thoracic Surgery (SEPAR) [4]. Appropriateness of empirical antimicrobial treatment in patients with known aetiology was defined when the isolated pathogens were susceptible in vitro to at least one of the antimicrobial agents administered.

Treatment failure was defined as clinical deterioration within $72 \mathrm{~h}$ of treatment resulting from one or more of the following causes: haemodynamic instability; appearance or impairment of respiratory failure; radiographic progression; or the appearance of new metastatic infectious foci (modified from the criteria used by MENENDEZ et al. [16]).

\section{Microbiological evaluation}

Regular sampling included sputum specimens, two blood cultures, urine samples for detection of Streptococcus pneumoniae (Binax Now S. pneumoniae Urinary Antigen Test; Emergo Europe, The Hague, the Netherlands) and Legionella pnemophila serogroup 1 (Binax Now L. pneumophila Urinary Antigen Test; Trinity Biotech, Bray, Ireland), and nasopharyngeal swabs for respiratory virus detection. Diagnosis of the following microorganisms was performed by means of paired serology at admission and during the third or sixth week thereafter: 1) atypical microorganisms, including L. pneumophila serogroup 1, Chlamydophila pneumoniae, Chlamydia psittaci, Mycoplasma pneumoniae and Coxiella burnetii; 2) respiratory viruses, i.e. influenza virus (A and B), parainfluenza virus (1, 2 and 3), respiratory syncytial virus and adenovirus. High titres of immunoglobulin (Ig)M antibodies in the serum during the acute phase was accepted for the diagnosis of atypical microorganisms, such as C. pneumoniae $(\geqslant 1: 32)$, C. burnetii $(\geqslant 1: 80)$ and $M$. pneumoniae (any positive titre).

\section{Diagnostic criteria}

The aetiology of pneumonia was classified as presumptive if a valid sputum sample yielded one or more predominant bacterial strain. The aetiology was considered definite if one of the following criteria was met: 1) blood culture yielding a bacterial or fungal pathogen (in the absence of an apparent extrapulmonary focus); 2) seroconversion (i.e. a four-fold increase in IgG titre for L. pneumophila $(\geqslant 1: 128)$, C. pneumoniae $\operatorname{IgG}(\geqslant 1: 512)$, C. psittaci IgG $(\geqslant 1: 64)$, C. burnetii $(\geqslant 1: 80)$ and respiratory viruses (i.e. influenza viruses $A$ and $B$, parainfluenza virus 1 to 3 , respiratory syncytial virus and adenovirus); 3) a single $\operatorname{IgM}$ titre for C. pneumoniae ( $\geqslant 1: 32$ ), C. burnetii $(\geqslant 1: 80)$ and $M$. pneumoniae (any titre); 4) a single titre $(\geqslant 1: 128)$ or a positive urinary antigen test for $L$. pneumophila serogroup $1 ; 5)$ a positive urinary antigen test for S. pneumoniae. For the purpose of this study, presumptive and definitive diagnoses were analysed together.

Strains were initially screened for susceptibility to antimicrobial agents using Sensititre (Trek Diagnostic Systems Ltd, East Grinstead, UK). Penicillin and other antibiotic susceptibilities were defined according to the 2008 breakpoints of the Clinical Laboratory Standards Institute (Wayne, PA, USA).

\section{Statistical analysis}

Categorical variables were described as frequencies and percentages. Continuous variables were expressed as means and standard deviations or medians and interquartile ranges for data that were not normally distributed (Kolmogorov-Smirnov test).

Categorical variables were compared with Fisher's exact test where appropriate. All tests were two-tailed and significance was set at $5 \%$. All analyses were performed with SPSS version 16.0 for Windows (SPSS Inc., Chicago, IL, USA).

\section{RESULTS}

\section{Patient characteristics}

During the study period, a total of 3,223 adult patients with CAP were admitted to our hospital; of these, 2,655 (82.4\%) were hospitalised and $568(17.6 \%)$ were treated as outpatients. The annual percentage of outpatients with CAP ranged between $9 \%$ and $23 \%$ during the study period (20002010). We analysed a total of 568 adult outpatients. There were $301(53.0 \%)$ males and $267(47.0 \%)$ females, with a mean age of $47.2 \pm 17.6 \mathrm{yrs} ; 110(19.4 \%)$ were aged $\geqslant 65$ yrs. We reexamined $550(97 \%)$ patients in ambulatory setting and contacted only $18(3 \%)$ patients by telephone. The main clinical characteristics and radiological findings are summarised in tables 1 and 2 . 


\section{TABLE 1 Clinical characteristics of outpatients}

$\begin{array}{lc}\text { Patients } \mathbf{n} & 568 \\ \text { Demographic } & \\ \text { Age yrs } & 47.2 \pm 17.6 \\ \quad \text { Age } \geqslant 65 \text { yrs } & 110(19.4) \\ \text { Male } & 301(53.0) \\ \text { Current smoking } & 214(37.9) \\ \text { Current alcohol abuse } & 71(12.6) \\ \text { Previous antibiotic } & 139(25.0) \\ \text { Influenza vaccine } & 97(19.6) \\ \text { Pneumococcal vaccine } & 25(5.1) \\ \text { Comorbidity } & 185(32.6) \\ \text { Chronic respiratory disease } & 125(22.0) \\ \text { Neurological disease } & 38(6.7) \\ \text { Diabetes mellitus } & 30(5.3) \\ \text { Chronic cardiovascular disease } & 15(2.6) \\ \text { Chronic liver disease } & 15(2.6) \\ \text { Chronic renal disease } & 4(0.7) \\ \text { Bacteraemia } & 21(5.8)^{\#} \\ \text { Multilobar infiltration } & 36(6.5) \\ \text { Pleural effusion } & 30(6.2) \\ \text { PSI } & \\ \text { I-III } & 551(97.0) \\ \text { IV } & 17(3.0) \\ \text { CURB-65 } & \\ \text { 0-1 } & 538(94.7) \\ \text { 2 } & 30(5.3) \\ \text { 30-day mortality } & 3(0.5)\end{array}$

Data are presented as mean \pm SD or $n(\%)$, unless otherwise stated. PSI: Pneumonia Severity Index; CURB-65: confusion of new onset, urea $>7 \mathrm{mmol} \cdot \mathrm{L}^{-1}$, respiratory rate $\geqslant 30$ breaths $\cdot \mathrm{min}^{-1}$, blood pressure $<90 \mathrm{mmHg}$ systolic or diastolic blood pressure $\leqslant 60 \mathrm{mmHg}$, and age $\geqslant 65 \mathrm{yrs}$. \# : percentage was calculated in patients with blood culture $(n=362)$.

A total of $139(25.0 \%)$ patients had received previous antimicrobial treatment prior to the emergency department visit as follows: $\beta$-lactams in 79 (56.8\%), fluoroquinolones in $24(17.3 \%)$, macrolides in $18(12.9 \%)$ and other antimicrobial agents in 18 $(12.9 \%)$ patients.

The distribution of groups by severity scores (PSI and CURB65) is shown in table 1 . In the emergency department, both scores placed most of the patients in the low-risk group (PSI 97.0\%; CURB-65 94.7\%).

\section{Microbial aetiology}

A complete or partial microbiological investigation was possible in $527(92.8 \%)$ patients while no microbiological test was performed in $41(7.2 \%)$. Aetiological diagnoses were established in $188(33.1 \%)$ cases. Of the $380(66.9 \%)$ patients without a defined aetiology, one-quarter $(25.0 \%)$ had received antimicrobial treatment in the previous 30 days.

As shown in tables 3 and 4 , the most frequent pathogens isolated were S. pneumoniae $(\mathrm{n}=66 ; 35.1 \%)$, M. pneumoniae $(\mathrm{n}=29 ; 15.4 \%)$, respiratory viruses $(\mathrm{n}=25 ; 13.3 \%)$, L. pneumophila $(\mathrm{n}=13 ; 6.9 \%)$, C. burnetii $(\mathrm{n}=11 ; 5.9 \%)$, C. pneumoniae

\begin{tabular}{|c|c|c|}
\hline TABLE 2 & \multicolumn{2}{|c|}{$\begin{array}{l}\text { Clinical presentation and laboratory data on } \\
\text { admission }\end{array}$} \\
\hline \multicolumn{2}{|l|}{ Patients n } & 568 \\
\hline \multicolumn{3}{|l|}{ Symptoms } \\
\hline \multicolumn{2}{|c|}{ Previous "common cold" symptoms } & $224(40.1)$ \\
\hline \multicolumn{2}{|l|}{ Fever } & 505 (88.9) \\
\hline \multicolumn{2}{|l|}{ Chills } & $314(56.6)$ \\
\hline \multicolumn{2}{|l|}{ Cough } & $454(80)$ \\
\hline \multicolumn{2}{|c|}{ Purulent sputum } & $300(52.8)$ \\
\hline \multicolumn{2}{|c|}{ Pleuritic pain } & $292(52.5)$ \\
\hline \multicolumn{2}{|l|}{ Dyspnoea } & $199(35.4)$ \\
\hline \multicolumn{2}{|c|}{ Nausea/vomiting } & $85(20.9)$ \\
\hline \multicolumn{3}{|c|}{ Radiographic features } \\
\hline \multicolumn{2}{|c|}{ Alveolar infiltrate pattern } & $510(89.8)$ \\
\hline \multicolumn{2}{|c|}{ Interstitial infiltrate pattern } & $40(7.0)$ \\
\hline \multicolumn{2}{|c|}{ Mixed infiltrate pattern } & $18(3.2)$ \\
\hline \multicolumn{2}{|c|}{ Pleural effusion } & $35(6.3)$ \\
\hline \multicolumn{2}{|l|}{ Atelectasis } & $15(2.7)$ \\
\hline \multicolumn{3}{|c|}{ Lobes affected } \\
\hline \multicolumn{2}{|l|}{1} & $532(93.7)$ \\
\hline \multicolumn{2}{|l|}{$\geqslant 2$} & $36(6.3)$ \\
\hline \multicolumn{3}{|l|}{ Vital signs } \\
\hline \multicolumn{2}{|c|}{ Respiratory rate breaths $\cdot \mathrm{min}^{-1}$} & $20.0 \pm 6.0$ \\
\hline \multicolumn{2}{|c|}{ Heart rate beats $\cdot \mathrm{min}^{-1}$} & $92.0 \pm 22.0$ \\
\hline \multicolumn{2}{|c|}{ Systolic blood pressure $\mathrm{mmHg}$} & $120.0 \pm 26.0$ \\
\hline \multicolumn{2}{|c|}{ Diastolic blood pressure $\mathrm{mmHg}$} & $72.0 \pm 12.0$ \\
\hline \multicolumn{3}{|c|}{ Laboratory findings median (IQR) } \\
\hline \multicolumn{2}{|c|}{ C-reactive protein $\mathrm{mg} \cdot \mathrm{dL}^{-1}$} & $12.2(15.4)$ \\
\hline \multicolumn{2}{|c|}{ Creatinine $\mathrm{mg} \cdot \mathrm{dL}^{-1}$} & $0.9(0.3)$ \\
\hline \multicolumn{2}{|c|}{ WBC count $\times 10^{9}$ cells $\cdot L^{-1}$} & $10.9(7.6)$ \\
\hline \multicolumn{2}{|c|}{ Platelet count $\times 10^{9}$ platelets $\cdot \mathrm{L}^{-1}$} & 248.5 (114.3) \\
\hline
\end{tabular}

Data are presented as $\mathrm{n}(\%)$ or mean $\pm \mathrm{SD}$, unless otherwise stated. IQR: interquartile range; WBC: white blood cell.

$(\mathrm{n}=10 ; 5.3 \%)$ and Haemophilus influenzae ( $\mathrm{n}=9 ; 4.8 \%)$ Unfortunately we did not record or investigate the possible source of C. burnetii infection in our series. More than one causative agent was found in $17(9.0 \%)$ patients and $S$. pneumoniae was the most frequent microorganism involved in mixed infections (14 out of $17 ; 82.4 \%$ ).

$21(3.7 \%)$ patients had bacteraemia (due to S. pneumoniae in 16 patients, H. influenzae in two, Streptococcus viridans in one, Escherichia coli in one and P. aeruginosa in one) (table 4). Patients with bacteraemia were re-evaluated when blood was drawn for culture in a daytime hospital setting to assess patients' clinical conditions (except for the case of $P$. aeruginosa bacteraemia that was lost at follow-up) and after 1 month in the outpatient clinic for follow-up. 34 isolates of $S$. pneumoniae were tested for susceptibility, with $30(88.2 \%)$ being susceptible to penicillin and four $(11.7 \%)$ resistant to penicillin. $28(82.3 \%)$ were sensitive to erythromycin and six $(17.6 \%)$ were resistant. Four $(11.7 \%)$ were sensitive to cefotaxime and one (2.9\%) was resistant.

Seven patients with positive blood cultures for S. pneumoniae also presented positive urinary antigen tests and three patients with positive pleural fluid also presented positive urinary antigen tests. 


\begin{tabular}{|c|c|c|c|}
\hline \multirow[t]{2}{*}{ TABLE 3} & \multicolumn{3}{|c|}{$\begin{array}{l}\text { Distribution of the causative microorganisms } \\
\text { identified in community-acquired pneumonia } \\
\text { outpatients }\end{array}$} \\
\hline & & $\begin{array}{c}\text { Total } \\
\text { population }\end{array}$ & $\begin{array}{l}\text { Population with } \\
\text { defined aetiology }\end{array}$ \\
\hline \multicolumn{2}{|l|}{ Patients n } & 568 & 188 \\
\hline \multicolumn{2}{|c|}{ Streptococcus pneumoniae } & $66(11.6)$ & $66(35.1)$ \\
\hline \multicolumn{2}{|c|}{ Streptococcus viridans } & $2(0.4)$ & $2(1.1)$ \\
\hline \multicolumn{2}{|c|}{ Staphylococcus aureus } & $1(0.2)$ & $1(0.5)$ \\
\hline \multicolumn{2}{|c|}{ Haemophilus influenzae } & $9(1.58)$ & $9(4.8)$ \\
\hline \multicolumn{2}{|c|}{ Escherichia coli } & $1(0.2)$ & $1(0.5)$ \\
\hline \multicolumn{2}{|c|}{ Pseudomonas aeruginosa } & $1(0.2)$ & $1(0.5)$ \\
\hline \multicolumn{2}{|l|}{ Others } & $3(0.5)$ & $3(1.6)$ \\
\hline \multicolumn{2}{|l|}{ Atypical } & $63(11.1)$ & $63(33.5)$ \\
\hline \multicolumn{2}{|c|}{ Legionella pneumophila } & $13(2.3)$ & $13(6.9)$ \\
\hline \multicolumn{2}{|c|}{ Mycoplasma pneumoniae } & $29(5.1)$ & $29(15.4)$ \\
\hline \multicolumn{2}{|c|}{ Coxiella burnetii } & $11(1.9)$ & $11(5.9)$ \\
\hline \multicolumn{2}{|c|}{ Chlamydophila pneumoniae } & $10(1.8)$ & $10(5.3)$ \\
\hline \multicolumn{2}{|c|}{ Respiratory virus } & $25(4.4)$ & 25 (13.3) \\
\hline \multicolumn{2}{|c|}{ Rhinovirus } & $6(1.1)$ & $6(24.0)$ \\
\hline \multicolumn{2}{|c|}{ Influenza virus A } & $14(2.5)$ & $14(56.0)$ \\
\hline \multicolumn{2}{|c|}{ Influenza virus B } & $2(0.4)$ & $2(8.0)$ \\
\hline \multicolumn{2}{|l|}{ Adenovirus } & $1(0.2)$ & $1(4.0)$ \\
\hline \multicolumn{2}{|c|}{ Respiratory syncytial virus } & $1(0.2)$ & $1(4.0)$ \\
\hline \multicolumn{2}{|c|}{ Parainfluenza virus 2} & $1(0.2)$ & $1(4.0)$ \\
\hline \multicolumn{2}{|l|}{ Mixed } & $17(3.0)$ & $17(9.0)$ \\
\hline \multicolumn{2}{|c|}{ S. pneumoniae + influenza A virus } & $9(1.6)$ & $9(52.9)$ \\
\hline \multicolumn{2}{|c|}{ H. influenzae + influenza virus A } & $2(0.4)$ & $2(11.7)$ \\
\hline \multicolumn{2}{|c|}{ S. pneumoniae $+\mathrm{H}$. influenzae } & $2(0.4)$ & $2(11.7)$ \\
\hline \multicolumn{2}{|c|}{ S. pneumoniae + adenovirus } & $1(0.2)$ & $1(5.8)$ \\
\hline \multicolumn{2}{|c|}{ S. pneumoniae $+P$. aeruginosa } & $1(0.2)$ & $1(5.8)$ \\
\hline \multicolumn{2}{|c|}{ S. pneumoniae + rhinovirus } & $1(0.2)$ & $1(5.8)$ \\
\hline \multicolumn{2}{|c|}{ L. pneumophila + rhinovirus } & $1(0.2)$ & $1(5.8)$ \\
\hline
\end{tabular}

Data are presented as $n(\%)$, unless otherwise stated.

The distribution of aetiology according to comorbidity is shown in table 5. Atypical pathogens, particularly M. pneumoniae, were more frequently present in patients without comorbidities, whereas S. pneumoniae and Legionella were more frequently present in patients with comorbidities. We also analysed the distribution of aetiologies according to smoking and alcohol habits, and the only significant result was that L. pneumophila and $H$. influenzae were more frequent in alcohol abusers (table S1a and b).

\section{Initial empirical antimicrobial treatment}

The initial antimicrobial treatment was fluoroquinolone monotherapy $(\mathrm{n}=315 ; 55.5 \%)$, a $\beta$-lactam plus a macrolide $(\mathrm{n}=133$; $23.4 \%), \beta$-lactam monotherapy $(n=40 ; 7.0 \%)$, fluoroquinolones plus a $\beta$-lactam $(n=34 ; 6.0 \%)$, macrolide monotherapy $(n=31$; $5.5 \%)$, a fluoroquinolone plus a macrolide $(n=11 ; 1.9 \%)$ or other combinations $(n=4 ; 0.7 \%)$. The initial empiric antimicrobial treatment followed the SEPAR guidelines in 496 (87.3\%) patients and was adequate according to the aetiology in 172 $(91.4 \%)$ out of 188 patients with a defined aetiology.

\section{Outcomes}

Mortality

The 30-day mortality was $0.5 \%(n=3)$ (two patients were readmitted to hospital due to pneumonia and one patient was readmitted for another cause). Two patients had comorbid chronic respiratory disease and another had acute leukaemia. The aetiology was established in two patients (Streptococcus viridans $(n=1)$ and adenovirus $(n=1))$. The complications in these patients were bacteraemia and multilobar infiltration. Empirical antibiotic treatment was inadequate in two patients. Patients who were re-admitted were treated in the respiratory intensive care unit and were not subject to treatment restrictions.

\section{Complications}

Pleural effusion as a pulmonary complication was observed in $30(5.3 \%)$ patients, with five $(16.6 \%)$ of these patients developing empyema due to $S$. pneumoniae $(\mathrm{n}=3)$ or $S$. viridans $(\mathrm{n}=2)$. All patients with empyema required re-admission. One patient was diagnosed with acute leukaemia during the first visit in the emergency department and discharged according to clinical stability and the patient's preference; unfortunately, the patient died later during the re-admission episode. Autopsy showed extensive pulmonary necrosis due to $S$. viridans. Most of the patients with pleural effusion were classified into risk classes I and II $(n=22 ; 73.3 \%)$ by PSI score and into risk class $0(n=19$; $63.3 \%$ ) by the CURB-65 score. None of the patients with a pulmonary complication demonstrated treatment failure.

None of the patients with bacteraemia $(n=21 ; 5.8 \%)$ was readmitted or died. $19(90.4 \%)$ of these patients received adequate empirical antibiotic treatment.

\section{Re-admissions}

$13(2.3 \%)$ patients were re-admitted to hospital within 30 days; nine $(69.2 \%)$ had comorbidities (chronic respiratory disease, neurological disease, diabetes mellitus, chronic cardiovascular disease and chronic liver disease). The PSI score placed these patients into risk classes II and III and the CURB-65 score into risk classes 0,1 and 2 .

The empirical antimicrobial treatment was adequate in $11(84.6 \%)$ of the patients who were re-admitted. Seven $(53.8 \%)$ patients were re-admitted because of respiratory problems (complicated pleural effusion, slow-responding pneumonia and chronic obstructive pulmonary disease exacerbation) and six (46.1\%) patients were readmitted for other causes not related to pneumonia. The mean time for readmission was 2 weeks, independent of the cause. Three patients died after re-admission.

\section{Treatment failure}

Treatment failure occurred in $13(2.3 \%)$ patients, being related to pneumonia in seven $(53.8 \%)$ patients and noninfectious in six $(46.1 \%)$. The PSI score classified the seven patients with infectious causes into risk classes I, II and III, while the CURB65 score placed these patients in risk classes 0,1 and 2 .

The microorganisms most frequently isolated in patients with infectious treatment failure were $M$. pneumoniae $(\mathrm{n}=3), \mathrm{S}$. pneumoniae $(\mathrm{n}=2)$ (both isolates being resistant to penicillin and erythromycin), $P$. aeruginosa plus $S$. pneumoniae $(n=1)$ and $S$. viridans $(n=1)$. Five out of the six cases of noninfectious treatment failure were patients who developed systemic complications after 
TABLE 4 Aetiology and diagnostic methods

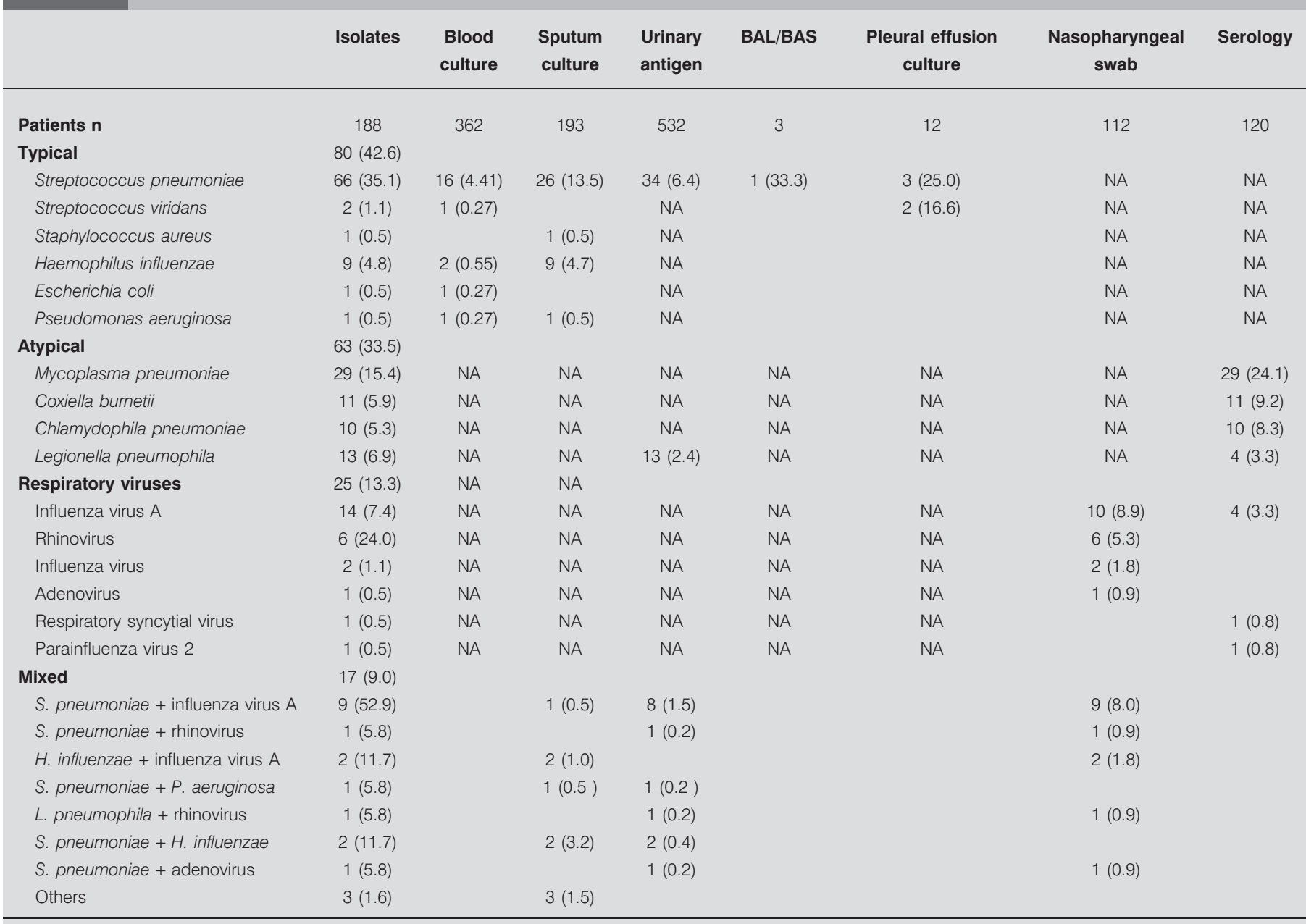

Data are presented as n (\%), unless otherwise stated. BAL: bronchoalveolar lavage; BAS: broncho-aspirate; NA: not applicable.

pneumonia (cardiac arrhythmia, endocarditis, pyelonephritis and acute renal failure) and two were hospitalised for other causes not related to the episode of pneumonia.

\section{Higher risk patients}

The PSI score classified 17 (3.0\%) patients into risk class IV. Five out of 17 patients had an aetiological diagnosis, the pathogens of these patients being S. pneumoniae, Staphylococcus aureus, L. pneumophila, respiratory virus and mixed aetiology (one case each). According to the SEPAR guidelines, $15(88.2 \%)$ patients received appropriate empirical antimicrobial treatment and according to their aetiologies, all patients were adequately treated. The CURB65 score did not assign patients to high-risk classes (3-5).

No patients considered as having higher risk according to PSI and CURB-65 required re-admission or died. Six (35.3\%) patients presented complications, i.e. multilobar infiltration $(n=4)$, pleural effusion $(n=1)$ and bacteraemia $(n=1)$.

\section{DISCUSSION}

The most important findings of our study are as follows. 1) Outpatients with CAP had a characteristic microbial pattern, with S. pneumoniae as the leading pathogen, followed by $M$. pneumoniae and respiratory viruses; L. pneumophila was present in $6.9 \%$ of cases with known aetiology. 2) Initial antimicrobial treatment was adequate according to guidelines in $87.3 \%$ and according to the aetiology in $91.4 \%$ of the cases. 3) The mortality rate was very low $(0.5 \%)$ and other adverse events were infrequent (complication rate $5.3 \%$, readmission rate $2.3 \%$ and treatment failure rate $2.3 \%$ ). 4) Deaths and adverse events occurred in low-risk patients but in not in the $3 \%$ of patients at higher risk according to the severity scores.

Several issues are crucial to adequately interpret studies on the aetiology of CAP in outpatients. First, it is very important to define the setting of treatment. On one hand, there are true primary care studies of patients referred to general practitioners [11, 17-19], while on the other there are hospital-based studies including patients initially examined at the emergency department $[10,12]$. Secondly, some studies are based on patients with "lower respiratory tract infections" [17, 18], not necessarily requiring a chest radiograph for confirmation of an infiltrate $[17,20]$, while others exclude patients without a new infiltrate [10-12]. In fact, these might be very different entities. In one 
TABLE 5 Distribution of aetiology according to comorbidity

\begin{tabular}{|c|c|c|c|c|c|c|c|c|c|}
\hline & $\begin{array}{c}\text { No } \\
\text { comorbidity }\end{array}$ & Comorbidity & $\begin{array}{l}\text { Chronic } \\
\text { respiratory } \\
\text { disease }\end{array}$ & $\begin{array}{c}\text { Neurological } \\
\text { disease }\end{array}$ & $\begin{array}{l}\text { Diabetes } \\
\text { mellitus }\end{array}$ & $\begin{array}{c}\text { Chronic } \\
\text { cardiovascular } \\
\text { disease }\end{array}$ & $\begin{array}{c}\text { Chronic } \\
\text { liver } \\
\text { disease }\end{array}$ & $\begin{array}{l}\text { Chronic } \\
\text { renal } \\
\text { disease }\end{array}$ & p-value \\
\hline Patients $\mathrm{n}$ & 383 & 185 & 122 & 36 & 31 & 15 & 14 & 4 & \\
\hline Typical & $46(12.0)$ & $34(18.4)$ & $26(21.3)$ & $6(16.6)$ & $7(22.6)$ & & $3(21.4)$ & & 0.053 \\
\hline Streptococcus pneumoniae & $38(9.9)$ & $28(15.1)$ & $20(16.3)$ & $5(13.8)$ & $5(16.1)$ & & $2(14.3)$ & & 0.093 \\
\hline Haemophilus influenzae & $6(1.6)$ & $3(1.6)$ & $3(2.5)$ & & $2(6.5)$ & & & & 1.00 \\
\hline Escherichia coli & & $1(0.5)$ & $1(0.8)$ & $1(2.7)$ & & & & & 0.33 \\
\hline Pseudomonas aeruginosa & $1(0.3)$ & & & & & & & & 1.00 \\
\hline Atypical & $49(12.8)$ & $14(7.6)$ & $9(7.4)$ & $3(8.3)$ & $1(3.2)$ & $1(6.7)$ & & & 0.065 \\
\hline Mycoplasma pneumoniae & $24(6.3)$ & $5(2.7)$ & $2(1.6)$ & $1(2.7)$ & $1(3.2)$ & $1(6.7)$ & & & 0.10 \\
\hline Influenza virus $A$ & $9(2.3)$ & $5(2.7)$ & $5(4.1)$ & & $1(3.2)$ & & $1(7.1)$ & & 0.78 \\
\hline Rhinovirus & $5(1.3)$ & $1(0.5)$ & $1(0.8)$ & $1(2.7)$ & & & & & 0.67 \\
\hline Influenza virus B & $2(0.5)$ & & & & & & & & 1.00 \\
\hline Adenovirus & & $1(0.5)$ & $1(0.8)$ & & & & & & 0.33 \\
\hline Respiratory syncytial virus & $1(0.3)$ & & & & & & & & 1.00 \\
\hline Parainfluenza virus 2 & & $1(0.5)$ & $1(0.8)$ & & $1(3.2)$ & & & & 0.33 \\
\hline Mixed & $12(3.1)$ & $5(2.7)$ & $2(1.6)$ & $2(5.6)$ & $1(3.2)$ & $1(6.7)$ & & & 1.00 \\
\hline S. pneumoniae + influenza virus A & $8(2.1)$ & $1(0.5)$ & $1(0.8)$ & $1(2.7)$ & & & & & 0.28 \\
\hline S. pneumoniae + rhinovirus & & $1(0.5)$ & & & $1(3.2)$ & & & & 0.33 \\
\hline H. influenzae + influenza virus A & $2(0.5)$ & & & & & & & & 1.00 \\
\hline S. pneumoniae $+H$. influenzae & & $2(1.1)$ & $1(0.8)$ & $1(2.7)$ & & & & & 0.11 \\
\hline S. pneumoniae + adenovirus & $1(0.3)$ & & & & & & & & 1.00 \\
\hline
\end{tabular}

Data are presented as $\mathrm{n}(\%)$, unless otherwise stated.

study including 364 adult patients with lower respiratory tract infections, pneumonia was radiologically verified in only 48 $(13 \%)$ patients [18]. However, due to the limited reliability of radiographic diagnosis of mild pneumonia [21], excluding patients without radiographic infiltrates may miss a proportion of patients with pneumonia. Finally, to date, few studies have included follow-up investigations in order to confirm the validity of CAP diagnosis and aetiology [22]. These differences are important, particularly because of the heavy impact of patient selection linked to the treatment setting. Our study is unique in that: 1) it is the only hospital-based study with patients being evaluated in an emergency department with high expertise in the treatment of acute respiratory tract infections, 2) all cases required a chest radiograph for confirmation of pneumonia, and 3) all survivors were re-evaluated within 30 days. This treatment setting clearly implies that only patients with confirmed CAP and carefully judged severity by both clinical means and severity tools were included [23]. Thus, the analysis presented is based on the most distinctive and valid data on outpatient CAP.
Several pitfalls must be recognised in studies evaluating the aetiology of CAP. The predefined set of diagnostic samples is usually incompletely recovered due to the many problems related to the retrieval of such samples, particularly with regard to sputum. For example, urinary antigens were not available the first years of our study. Antimicrobial pretreatment clearly reduces and characteristically affects the diagnostic yield of culture-based investigations [24]; in fact, of those without aetiology defined in our study, $72.6 \%$ had received previous antimicrobial treatment. Thus, relating aetiologies to the total population underestimates the incidence, whereas the reverse is true when these are related to the population with a defined aetiology. We provide both ratios and argue that the true incidence might be anywhere within these two. Another important potential bias is the presence of comorbidities which might affect the resulting microbial pattern. Therefore, we provided an analysis of aetiology in relation to comorbidity and found a trend for S. pneumoniae to occur more frequently in comorbid patients and M. pneumoniae in patients without comorbidity. 
Overall, S. pneumoniae was the most common pathogen, followed by $M$. pneumoniae and respiratory viruses. This is comparable to other studies [11, 19, 20]. Several authors have reported a high frequency of $M$. pneumonia [10, 12], even being more frequent than S. pneumoniae in outpatients [12]. In fact, age is the main host factor related to the incidence of $M$. pneumoniae [25] and the mean age of the patients in this series was remarkably low. Regardless of the differences reported, initial anti-pneumococcal coverage remains mandatory. L. pneumophila was the cause of CAP in $6.9 \%$ of cases. This finding is important for empirical antibiotic treatment in our setting.

Establishing the aetiology only marginally (by $4.1 \%$ ) increased the proportion of patients receiving adequate treatment as compared with those treated according to guidelines. In addition, an established aetiology was obviously not crucial in managing patients with adverse outcomes. However, a small but relevant number of patients had resistance to penicillin $(n=4 ; 11.7 \%$ of isolates tested) and two cases had unexpected pathogens (E. coli and P. aeruginosa). Moreover, nine out of 13 patients with mild L. pneumonia were not adequately treated, which might cause prolonged morbidity. Patients requiring short-term observation in an emergency care unit and/or presenting with pleural effusion and/or significant comorbidity might be candidates for microbial investigation, particularly for pyogenic pathogens and L. pneumophila.

Overall, mortality and other adverse outcomes were rare. In line with previous reports, pleural effusion $(16.6 \%$ progressed to empyema) was the most frequent cause of complications [26]. Interestingly, bacteraemia had no impact on any outcome measure. Re-admission was mostly related to comorbidity (in $69.2 \%$ of cases) and, actually, was the only cause of re-admission in roughly half of these patients. Treatment failure was rare $(2.3 \%)$ and only half of the cases were related to infection. Again, comorbidity was the main driver behind this adverse outcome. These outcomes compare favourably to other reports [11, 22].

Both severity scores correctly identified the patients at low risk in the vast majority of cases. None of the cases at increased risk according to the severity scores had an adverse outcome. Thus, the few patients dying and experiencing other adverse outcomes were all in the low-risk group. Our data indicate that a closer view on patients with pleural effusions and comorbidities might reduce underestimations of risks according to clinical judgment and severity scores. It has previously been shown that ambulatory treatment in low-risk patients is equivalent to hospitalisation in the absence of respiratory failure, unstable comorbidity, pleural effusions and social problems [22].

In conclusion, most patients managed as outpatients after presenting in a hospital emergency department can be safely treated based on the current guidelines. Antimicrobial pneumococcal coverage in all patients remains crucial. L. pneumophila cannot be disregarded. Patients presenting with pleural effusions and/or comorbidities should probably be more closely observed if not admitted to hospital.

\section{SUPPORT STATEMENT}

The study was supported by 2009 Support to Research Groups of Catalunya grant 911, Ciber de Enfermedades Respiratorias (CibeRes CB06/06/0028) and Institut de Investigaciones Biomédicas August Pi i Sunyer.

\section{STATEMENT OF INTEREST}

A statement of interest for A. Torres can be found at www.erj. ersjournals.com $/$ site $/ \mathrm{misc} /$ statements.xhtml

\section{REFERENCES}

1 Niederman MS. Recent advances in community-acquired pneumonia: inpatient and outpatient. Chest 2007; 131: 1205-1215.

2 Lim WS, Baudouin SV, George RC, et al. BTS guidelines for the management of community acquired pneumonia in adults: update 2009. Thorax 2009; 64: Suppl. 3, iii1-iii55.

3 Mandell LA, Wunderink RG, Anzueto A, et al. Infectious Diseases Society of America/American Thoracic Society consensus guidelines on the management of community-acquired pneumonia in adults. Clin Infect Dis 2007; 44: Suppl. 2, S27-S72.

4 Menendez R, Torres A, Aspa J, et al. Neumonía adquirida en la comunidad. Nueva normativa de la Sociedad Española de Neumología y Cirugía Torácica (SEPAR) [Community acquired pneumonia. New guidelines of the Spanish Society of Chest Diseases and Thoracic Surgery (SEPAR)]. Arch Bronconeumol 2010; 46: 543-558.

5 Chalmers JD, Akram AR, Hill AT. Increasing outpatient treatment of mild community-acquired pneumonia: systematic review and meta-analysis. Eur Respir J 2011; 37: 858-864.

6 Cilloniz C, Ewig S, Polverino E, et al. Microbial aetiology of community-acquired pneumonia and its relation to severity. Thorax 2011; 66: 340-346.

7 Almirall J, Boixeda R, Bolibar I, et al. Differences in the etiology of community-acquired pneumonia according to site of care: a population-based study. Respir Med 2007; 101: 2168-2175.

8 Fine MJ, Auble TE, Yealy DM, et al. A prediction rule to identify low-risk patients with community-acquired pneumonia. $N$ Engl J Med 1997; 336: 243-250.

9 Lim WS, van der Eerden MM, Laing R, et al. Defining community acquired pneumonia severity on presentation to hospital: an international derivation and validation study. Thorax 2003; 58: 377-382.

10 Marrie TJ, Peeling RW, Fine MJ, et al. Ambulatory patients with community-acquired pneumonia: the frequency of atypical agents and clinical course. Am J Med 1996; 101: 508-515.

11 Bochud PY, Moser F, Erard P, et al. Community-acquired pneumonia. A prospective outpatient study. Medicine (Baltimore) 2001; 80: 75-87.

12 Miyashita N, Fukano H, Mouri K, et al. Community-acquired pneumonia in Japan: a prospective ambulatory and hospitalized patient study. J Med Microbiol 2005; 54: 395-400.

13 Yealy DM, Auble TE, Stone RA, et al. Effect of increasing the intensity of implementing pneumonia guidelines: a randomized, controlled trial. Ann Intern Med 2005; 143: 881-894.

14 Almirall J, Gonzalez CA, Balanzo X, et al. Proportion of communityacquired pneumonia cases attributable to tobacco smoking. Chest 1999; 116: 375-379.

15 de Roux A, Cavalcanti M, Marcos MA, et al. Impact of alcohol abuse in the etiology and severity of community-acquired pneumonia. Chest 2006; 129: 1219-1225.

16 Menendez R, Torres A, Zalacain R, et al. Risk factors of treatment failure in community acquired pneumonia: implications for disease outcome. Thorax 2004; 59: 960-965.

17 Creer DD, Dilworth JP, Gillespie SH, et al. Aetiological role of viral and bacterial infections in acute adult lower respiratory tract infection (LRTI) in primary care. Thorax 2006; 61: 75-79.

18 Holm A, Nexoe J, Bistrup LA, et al. Aetiology and prediction of pneumonia in lower respiratory tract infection in primary care. $\mathrm{Br}$ J Gen Pract 2007; 57: 547-554.

19 Macfarlane JT, Colville A, Guion A, et al. Prospective study of aetiology and outcome of adult lower-respiratory-tract infections in the community. Lancet 1993; 341: 511-514. 
20 Woodhead MA, Macfarlane JT, McCracken JS, et al. Prospective study of the aetiology and outcome of pneumonia in the community. Lancet 1987; 1: 671-674.

21 Albaum MN, Hill LC, Murphy M, et al. Interobserver reliability of the chest radiograph in community-acquired pneumonia. PORT Investigators. Chest 1996; 110: 343-350.

22 Carratala J, Fernandez-Sabe N, Ortega L, et al. Outpatient care compared with hospitalization for community-acquired pneumonia: a randomized trial in low-risk patients. Ann Intern Med 2005; 142: 165-172.

23 Choudhury G, Chalmers JD, Mandal P, et al. Physician judgment is a crucial adjunct to pneumonia severity scores in low risk patients. Eur Respir J 2011; 38: 643-648.
24 van de Garde EM, Souverein PC, van den Bosch JM, et al. Prior outpatient antibacterial therapy as prognostic factor for mortality in hospitalized pneumonia patients. Respir Med 2006; 100: 1342-1348.

25 Carr B, Walsh JB, Coakley D, et al. Prospective hospital study of community acquired lower respiratory tract infection in the elderly. Respir Med 1991; 85: 185-187.

26 Roson B, Carratala J, Dorca J, et al. Etiology, reasons for hospitalization, risk classes, and outcomes of communityacquired pneumonia in patients hospitalized on the basis of conventional admission criteria. Clin Infect Dis 2001; 33: 158-165. 